PROCEEDINGS OF THE

AMERICAN MATHEMATICAL SOCIETY

Volume 131, Number 4, Pages 1215-1224

S 0002-9939(02)06637-6

Article electronically published on July 26, 2002

\title{
ON THE FIRST TWO EIGENVALUES OF STURM-LIOUVILLE OPERATORS
}

\author{
MIKLÓS HORVÁTH
}

(Communicated by Carmen C. Chicone)

\begin{abstract}
Among the Schrödinger operators with single-well potentials defined on $(0, \pi)$ with transition point at $\frac{\pi}{2}$, the gap between the first two eigenvalues of the Dirichlet problem is minimized when the potential is constant. This extends former results of Ashbaugh and Benguria with symmetric singlewell potentials. An analogous result is given for the Dirichlet problem of vibrating strings with single-barrier densities for the ratio of the first two eigenvalues.
\end{abstract}

\section{INTRODUCTION}

Consider the Dirichlet problem for the Schrödinger operator acting on $(0, \pi)$ :

$$
\begin{aligned}
-y "+V(x) y & =\lambda y \quad \text { on } \quad[0, \pi], \\
y(0)=y(\pi) & =0 .
\end{aligned}
$$

Let $0 \leqslant a \leqslant \pi$ be fixed. Following Ashbaugh and Benguria 4 we call the function $V$ a single-well function if $V$ is decreasing in $[0, a]$ and increasing in $[a, \pi]$. Analogously $V$ is called a single-barrier function if it is increasing in $[0, a]$ and decreasing in $[a, \pi]$. The point $a$ is the transition point in both cases.

Ashbaugh and Benguria proved in 4 that if $V$ is a symmetric single-well potential, then the first two eigenvalues of (1)-(2) satisfy

$$
\lambda_{2}-\lambda_{1} \geqslant 3
$$

with equality if and only is $V$ is constant. They formulated the conjecture that the hypothesis on the symmetry of $V$ should be somehow eliminated, e.g. (3) should be true for (nonsymmetric) convex potentials. This conjecture was later proved by Lavine [10]. Our first goal is to investigate another natural way to remove symmetry. We show that (3) still holds for nonsymmetric single-well potentials if the transition point remains the midpoint:

Theorem 1.1. Let $V$ be a (not necessarily symmetric) single-well potential on $[0, \pi]$ with transition point $a=\frac{\pi}{2}$. Then the first two eigenvalues of the Dirichlet problem (1)-(2) satisfy (3) with equality if and only if $V$ is constant. If $a \neq \frac{\pi}{2}$, there are single-well potentials $V$ with $\lambda_{2}-\lambda_{1}<3$.

Received by the editors July 25, 2001 and, in revised form, November 18, 2001.

2000 Mathematics Subject Classification. Primary 34L15, 34B25.

Supported by the Hungarian Grant OTKA T032374.

(C)2002 American Mathematical Society 
Remark 1.2. It is also proved in [4] that the reversed inequality of (3) holds for symmetric single-barrier potentials. However this result cannot be extended to any class of nonsymmetric single-barrier potentials with fixed transition point, including fixing it at the midpoint. Counterexamples are the stepfunctions mentioned at the end of the proof of Theorem 1.1.

Recently, M-J. Huang [7] gave the statements corresponding to the above results of [4] and of Lavine for the case of vibrating strings. More precisely, consider the Dirichlet problem

$$
\begin{aligned}
& u^{\prime \prime}+\lambda \varrho(x) u=0 \quad \text { in }[0, \pi], \\
& u(0)=u(\pi)=0,
\end{aligned}
$$

for the vibrating string; here the density function $\varrho(x)$ is supposed to be positive. Huang proved that in the class of concave densities or symmetric single-barrier densities the first two eigenvalues of (4)-(5) satisfy

$$
\frac{\lambda_{2}}{\lambda_{1}} \geqslant 4
$$

and in the class of symmetric single-well densities we have

$$
\frac{\lambda_{2}}{\lambda_{1}} \leqslant 4
$$

In (6) resp. (7) equality occurs if and only if $\varrho$ is constant. Here we can also drop the condition of symmetry:

Theorem 1.3. Let $\varrho$ be a (not necessarily symmetric) single-barrier density function on $[0, \pi]$ with transition point $a=\frac{\pi}{2}$. Then the first two eigenvalues of the Dirichlet problem (4)-(5) satisfy (6) with equality if and only if $\varrho$ is constant. If the transition point $a \neq \frac{\pi}{2}$, there are single-barrier densities @ for which $\frac{\lambda_{2}}{\lambda_{1}}<4$.

Remark 1.4. Theorem 1.3 is not true with single-barrier replaced by single-well, (6) replaced by (7) and with anywhere fixed transition point; counterexamples are given at the end of the proof.

Finally we mention some papers related to these topics. In [2] Ashbaugh and Benguria proved (7) for the Dirichlet problem of Schrödinger operators with nonnegative potentials. Generalizations for other eigenvalue ratios (e.g. $\left.\frac{\lambda_{n}}{\lambda_{1}} \leqslant n^{2}\right)$ are given in [3]. These results are then extended in 5] for general Sturm-Liouville operators; see also Y-L. Huang and Law 8]. Most of these results provide upper bounds for some eigenvalue ratios. Worse lower bounds for $\frac{\lambda_{2}}{\lambda_{1}}$ than (6) for a larger class of densities than in Theorem 1.2 above are given in [5]. For other lower estimates see Gentry and Banks 6], Keller 9 and Mahar and Willner 11. The first gap of eigenvalues for some symmetric double-well situations is investigated in Abramovich [1].

The proofs of Theorems 1.1 and 1.3 consist of two parts. First we reduce the problem to the search of minimum among the stepfunction potentials (or densities), being constant in $\left(0, \frac{\pi}{2}\right)$ and $\left(\frac{\pi}{2}, \pi\right)$. In such cases the eigenfunctions can be expressed by trigonometric functions, so finding the minimum of $\lambda_{2}-\lambda_{1}$ (or $\frac{\lambda_{2}}{\lambda_{1}}$ ) requires the study of nonlinear equations containing the tangent functions. This is given in Section 2, while in Section 3 we finish the proofs of Theorems 1.1 and 1.3. The use of trigonometric functions appears in several papers, e.g. in [8], [3], [11], [9], [5]. 


\section{Solutions of trigonometric EQUATions}

In this part of the paper we prove some statements necessary for later purposes. In the proof of Theorem 1.1 we need

Lemma 2.1. Define $f(t)=\sqrt{t} \cot \left(\sqrt{t} \frac{\pi}{2}\right)$ for real $t$ and let $m>0$. Then the first two real solutions of the equation $f(t)=-f(t-m)$ satisfy

$$
t_{2}-t_{1}>3 \text {. }
$$

Proof. Step 1. The function $f(t)$ is strictly decreasing in the intervals $(-\infty, 4)$, $(4,16)$ and in general in $\left(4 n^{2}, 4(n+1)^{2}\right), n \geqslant 1$. This follows from the formulae

$$
\begin{aligned}
& f^{\prime}(t)=\frac{\sin (\sqrt{t} \pi)-\sqrt{t} \pi}{4 \sqrt{t} \sin ^{2}\left(\sqrt{t} \frac{\pi}{2}\right)}<0 \text { for } t>0, \quad t \neq 4 n^{2}, \\
& f^{\prime}(t)=\frac{\sqrt{-t} \pi-\sinh (\sqrt{-t} \pi)}{4 \sqrt{-t} \sinh ^{2}\left(\sqrt{-t} \frac{\pi}{2}\right)}<0 \text { for } t<0 .
\end{aligned}
$$

The monotonity then implies that

$$
t_{1} \in(1,4), t_{2} \in(4,16), f\left(t_{1}\right)<0 \text {. }
$$

Step 2. The functions $t_{1}(m)$ and $t_{2}(m)$ are strictly increasing functions of $m>0$. Indeed, if $t(m)$ denotes any of the solutions of $f(t)=-f(t-m)$, continuous in $m$, then

$$
\begin{aligned}
f(t(m)) & =-f(t(m)-m)>-f(t(m)-m-\Delta m), \\
f(t(m+\Delta m)) & =-f(t(m+\Delta m)-m-\Delta m)
\end{aligned}
$$

implies $t(m+\Delta m)>t(m)$.

Step 3. We can suppose

$$
0<m<8, \quad 4<t_{2}<9, \quad f\left(t_{2}\right)>0 .
$$

Indeed, for $m=8$ we have $t_{2}=9$, so $m \geqslant 8$ implies $t_{2} \geqslant 9$, whence $t_{2}-t_{1}>5>3$. From $m<8$ we get that $t_{2}<9$ and consequently $f\left(t_{2}\right)>0$.

Step 4. The statement of Lemma 2.1 will follow from the estimates

$$
\frac{d t_{1}}{d m}<\frac{1}{2}<\frac{d t_{2}}{d m} \quad \text { for } \quad 0<m<8
$$

since for $m=0$ we have $t_{2}-t_{1}=3$. To verify (10) we use the formula

$$
\frac{1-t_{i}^{\prime}(m)}{t_{i}^{\prime}(m)}=\frac{f^{\prime}\left(t_{i}(m)\right)}{f^{\prime}\left(t_{i}(m)-m\right)}, \quad i=1,2,
$$

which is obtained by differentiating $f\left(t_{i}(m)\right)=-f\left(t_{i}(m)-m\right)$ in $m$. Since $f^{\prime}$ is negative, (10) is equivalent to

$$
f^{\prime}\left(t_{1}-m\right)>f^{\prime}\left(t_{1}\right)
$$

and

$$
f^{\prime}\left(t_{2}-m\right)<f^{\prime}\left(t_{2}\right)
$$

Step 5 . We show $\left(12^{\prime}\right)$. We apply the formula

$$
f^{\prime}(t)=\frac{1}{2 \sqrt{t}} \cot \left(\sqrt{t} \frac{\pi}{2}\right)-\frac{\pi}{4}\left(1+\cot ^{2}\left(\sqrt{t} \frac{\pi}{2}\right)\right)
$$


and express $\cot \left(\sqrt{t-m} \frac{\pi}{2}\right)$ from $f(t)=-f(t-m)$ to obtain

$$
\begin{aligned}
& f^{\prime}\left(t_{2}-m\right)-f^{\prime}\left(t_{2}\right) \\
= & \frac{1}{\sqrt{t_{2}}} \frac{1}{t_{2}-m} \cot \left(\sqrt{t_{2}} \frac{\pi}{2}\right) \cdot\left[\frac{m-2 t_{2}}{2}-\frac{\pi}{4} m f\left(t_{2}\right)\right] .
\end{aligned}
$$

We know $t_{2}>1+m$ since for $t=1+m$ we have $-f(t-m)=0$ and $-f\left(t_{2}-m\right)=$ $f\left(t_{2}\right)>0$. So $t_{2}-m>0, f\left(t_{2}\right)>0, m-2 t_{2}<0$ and (14) proves indeed (12').

Step 6 . We prove (12) by showing that

$$
f^{\prime}\left(t_{1}-m\right)>-\frac{\pi}{4}
$$

and

$$
f^{\prime}\left(t_{1}\right)<-\frac{\pi}{4}
$$

Applying (13) again we get

$$
f^{\prime}\left(t_{1}\right)+\frac{\pi}{4}=\frac{1}{2 \sqrt{t_{1}}} \cot \left(\sqrt{t_{1}} \frac{\pi}{2}\right)-\frac{\pi}{4} \cot ^{2}\left(\sqrt{t_{1}} \frac{\pi}{2}\right)<0
$$

since $f\left(t_{1}\right)<0$. This proves $\left(15^{\prime}\right)$. To show (15) we again express $\cot \left(\sqrt{t_{1}-m} \frac{\pi}{2}\right)$ from $f\left(t_{1}\right)=-f\left(t_{1}-m\right)$ and we get

$$
f^{\prime}\left(t_{1}-m\right)+\frac{\pi}{4}=\frac{-f\left(t_{1}\right)}{2} \frac{1}{t_{1}-m}\left[1+\frac{\pi}{2} f\left(t_{1}\right)\right] .
$$

Analyzing the sign of the right-hand side of (16) we remark first that $t_{1}-m$ is positive for $m=0$ and negative for $m=8$ so there must be a value $m^{*}$ with $t_{1}\left(m^{*}\right)=m^{*}$. For such values $m^{*}$ we have $1+\frac{\pi}{2} f\left(t_{1}\left(m^{*}\right)\right)=0$ since the derivative in (16) must be finite. But $t_{1}$ is strictly increasing in $m, 1+\frac{\pi}{2} f\left(t_{1}\right)$ is strictly decreasing in $m$ so there is only one value $m^{*}$, and

$$
\begin{array}{llll}
t_{1}-m>0, & 1+\frac{\pi}{2} f\left(t_{1}\right)>0 & \text { if } & 0<m<m^{*}, \\
t_{1}-m<0, & 1+\frac{\pi}{2} f\left(t_{1}\right)<0 & \text { if } & m^{*}<m<8 .
\end{array}
$$

With $f\left(t_{1}\right)<0$ this implies (15). So $(12),\left(12^{\prime}\right)$ and then (10) is verified; the proof of Lemma 2.1 is complete.

In proving Theorem 1.3 we need

Lemma 2.2. Let $m>1$. Then the first two positive solutions of $f(\lambda)=-f(\lambda m)$ satisfy $\frac{\lambda_{2}}{\lambda_{1}}>4$. As solutions we allow values $\lambda$ for which $f(\lambda)$ and $f(\lambda m)$ are infinite; $f(\lambda)$ is defined in Lemma 2.1.

Proof. Again we argue in steps. By the substitutions $t=\sqrt{\lambda} \frac{\pi}{2}, d=\sqrt{m}$ the equation $f(\lambda)=-f(\lambda m)$ can be transformed into the form

$$
\tan t=-\frac{1}{d} \tan (t d)
$$

We have to show that the first two solutions $t_{1}, t_{2}$ satisfy $t_{2}>2 t_{1}$ if $d>1$.

Step 1. If $d>1$ is increasing, then $t_{1}$ decreases and $t_{1} d$ increases. To see this we first remark that $\frac{\pi}{2 d}<t_{1}<\min \left(\frac{3 \pi}{2 d}, \frac{\pi}{2}\right)$. On the other hand, $\frac{\partial}{\partial d}\left[\frac{1}{d} \tan \left(t_{1} d\right)\right]=$ $\frac{2 t_{1} d-\sin 2 t_{1} d}{2 d^{2} \cos ^{2}\left(t_{1} d\right)}>0$ shows that $-\frac{1}{d} \tan \left(t_{1} d\right)$ strongly decreases in $d$. Hence for $d<d^{\prime}$

$$
\tan t_{1}=-\frac{1}{d} \tan \left(t_{1} d\right)>-\frac{1}{d^{\prime}} \tan \left(t_{1} d^{\prime}\right), \tan t_{1}^{\prime}=-\frac{1}{d^{\prime}} \tan \left(t_{1}^{\prime} d^{\prime}\right)
$$


so $t_{1} \leqslant t_{1}^{\prime}$ would lead to a contradiction:

$$
\tan t_{1}>-\frac{1}{d^{\prime}} \tan \left(t_{1} d^{\prime}\right) \geqslant-\frac{1}{d^{\prime}} \tan \left(t_{1}^{\prime} d^{\prime}\right)=\tan t_{1}^{\prime} \geqslant \tan t_{1}
$$

The monotonity of $t_{1} d$ is analogously derived from the fact that $\tau=t_{1} d$ is the first positive solution of $\tan \tau=-d \tan \frac{\tau}{d}$.

Step 2. For $1<d \leqslant 3$ we have $t_{1}<\frac{3 \pi}{4 d}$. Since $t_{1} d$ is increasing, it is enough to check it for $d=3$. In this case $t_{2}=\frac{\pi}{2}$ and $t_{1} \in\left(\frac{\pi}{6}, \frac{\pi}{2}\right)$ is the solution of $\tan t_{1}=$ $-\frac{1}{3} \tan \left(3 t_{1}\right)=\frac{\frac{1}{3} \tan ^{3} t_{1}-\tan t_{1}}{1-3 \tan ^{2} t_{1}}$. Solving this equality we get $\tan t_{1}=\sqrt{\frac{3}{5}}<1$, hence $t_{1}<\frac{\pi}{4}=\frac{3 \pi}{4 d}$.

Step 3. For $1<d \leqslant 3$ we have $t_{2}>2 t_{1}$. We have seen this for $d=3$. If $d<3$, then $t_{1}, t_{2} \in\left(\frac{\pi}{2 d}, \frac{3 \pi}{2 d}\right)$ and $t_{2}>\frac{\pi}{2}$. Hence if $t_{1} \leqslant \frac{\pi}{4}$, then $t_{2}>2 t_{1}$, only the case $t_{1}>\frac{\pi}{4}$ remains, when (by Step 2) $2 t_{1}, t_{2} \in\left(\frac{\pi}{2}, \frac{3 \pi}{2 d}\right)$. On this interval tan $t$ and $-\frac{1}{d} \tan (t d)$ are strongly monotonous, hence

$$
t_{2}>2 t_{1} \Leftrightarrow \tan \left(2 t_{1}\right)<-\frac{1}{d} \tan \left(2 t_{1} d\right)
$$

We solve the inequality on the right by applying the formulae $\tan (2 \alpha)=\frac{2 \tan \alpha}{1-\tan ^{2} \alpha}$, $\tan t_{1}=-\frac{1}{d} \tan \left(t_{1} d\right)$ as follows:

$$
\frac{2 \tan t_{1}}{1-\tan ^{2} t_{1}}<-\frac{1}{d} \frac{-2 d \tan t_{1}}{1-d^{2} \tan ^{2} t_{1}}
$$

From $\tan t_{1}>0$ we finally get

$$
\frac{1}{1-\tan ^{2} t_{1}}<\frac{1}{1-d^{2} \tan ^{2} t_{1}} .
$$

Since $t_{1}>\frac{\pi}{4}$, the denominator on the left is negative and then the other denominator is also negative. Thus (18) means $\tan ^{2} t_{1}<d^{2} \tan ^{2} t_{1}$ which is of course valid. This proves by (17) the statement of Step 3 .

Step 4. For $d>3$ we also have $t_{2}>2 t_{1}$. To see this, we first observe that $t_{1}, t_{2} \in\left(0, \frac{\pi}{2}\right), t_{1}, \in\left(\frac{\pi}{2 d}, \frac{3 \pi}{2 d}\right), t_{2} \in\left(\frac{3 \pi}{2 d}, \frac{5 \pi}{2 d}\right)$. If $2 t_{1} \leqslant \frac{3 \pi}{2 d}$, then $t_{2}>2 t_{1}$ follows from $t_{2}>\frac{3 \pi}{2 d}$, so we can suppose $2 t_{1}>\frac{3 \pi}{2 d}$. Since $t_{1}<\frac{\pi}{4}$ for $d=3$ and $t_{1}$ is decreasing in $d, t_{1}<\frac{\pi}{4}$ still holds for $d>3$, too. Consequently we have $2 t_{1}, t_{2} \in\left(\frac{3 \pi}{2 d}, \frac{\pi}{2}\right)$. On this interval $\tan t$ and $-\frac{1}{d} \tan (t d)$ are strongly monotonic, so we can state (17) and reduce its right inequality to the form (18). From $\tan t_{1}=-\frac{1}{d} \tan \left(t_{1} d\right)<0$ we get $t_{1} d<\pi$; with $t_{1} d>\frac{3 \pi}{4}$ this means that $d^{2} \tan ^{2} t_{1}=\tan ^{2}\left(t_{1} d\right)<1$. Thus the denominators in (18) are positive and then (18) reduces to the trivial inequality $\tan ^{2} t_{1}<d^{2} \tan ^{2} t_{1}$. This proves Step 4 and the entire Lemma 2.2.

\section{The Proof of Theorems 1.1 And 1.3}

We recall some known properties of the eigenfunctions of the Dirichlet problem (1)-(2), given either in Lavine [10] or in classical monographs on eigenvalue problems. We norm the eigenfunctions $y_{n}$ such that $y_{n}(x)$ is positive for small $x>0$ and

$$
\int_{0}^{\pi} y_{n}^{2}=1
$$


Then $y_{1}$ is positive in $(0, \pi) ; y_{2}$ has an inner root $0<x_{0}<\pi$ and is positive in $\left(0, x_{0}\right)$ and negative in $\left(x_{0}, \pi\right)$. Furthermore there exist two values $0 \leqslant x_{-}<x_{0}<x_{+} \leqslant \pi$ such that

$$
y_{2}^{2}-y_{1}^{2}\left\{\begin{array}{lll}
>0 & \text { on } & \left(0, x_{-}\right) \cup\left(x_{+}, \pi\right), \\
<0 & \text { on } \quad\left(x_{-}, x_{+}\right),
\end{array}\right.
$$

and both sets in (20) are nonempty. Recall the following known formula for the derivatives of the eigenvalues. Let $V_{0}, V_{1}$ be single-well potentials and

$$
V(x, t)=t V_{1}(x)+(1-t) V_{0}(x) .
$$

Then the derivative of $\lambda_{n}$ with respect to $t$ is

$$
\dot{\lambda}_{n}=\int_{0}^{\pi} \dot{V} y_{n}^{2}=\int_{0}^{\pi}\left(V_{1}(x)-V_{0}(x)\right) y_{n}^{2}(x, t) d x,
$$

hence

$$
\left(\lambda_{2}-\lambda_{1}\right)=\int_{0}^{\pi}\left(V_{1}(x)-V_{0}(x)\right)\left(y_{2}^{2}(x, t)-y_{1}^{2}(x, t)\right) d t .
$$

Proof of Theorem 1.1. Denote for $M>0$

$$
A_{M}=\left\{V: 0 \leqslant V \leqslant M, V \text { is single-well with transition point at } \frac{\pi}{2}\right\} .
$$

It is known that there exists an optimal potential $V_{0} \in A_{M}$ giving the minimal value $\lambda_{2}-\lambda_{1}$ over $A_{M}$. Therefore it is enough to show that for large $M$ the optimal $V_{0}$ must be constant since every single-well potential with transition point $\frac{\pi}{2}$ can be shifted into $A_{M}$ if $M$ is large. Consider some cases:

A) $x_{-} \leqslant \frac{\pi}{2}<x_{+}$(the case $\frac{\pi}{2}=x_{+}$is similar). Let

$$
V_{1}(x)=\left\{\begin{array}{lll}
V_{0}\left(x_{-}\right) & \text {on } & \left(0, \frac{\pi}{2}\right), \\
V_{0}\left(x_{+}\right) & \text {on } & \left(\frac{\pi}{2}, \pi\right) ;
\end{array}\right.
$$

we see that $V_{1} \in A_{M}$ and

$$
V_{1}-V_{0}\left\{\begin{array}{lll}
\leqslant 0 & \text { on } & \left(0, x_{-}\right) \cup\left(x_{+}, \pi\right), \\
\geqslant 0 & \text { on } & \left(x_{-}, x_{+}\right) .
\end{array}\right.
$$

Define

$$
V(x, t)=t V_{1}(x)+(1-t) V_{0}(x) ;
$$

it belongs to $A_{M}$ if $0 \leqslant t \leqslant 1$. Thus by the optimality of $V_{0}$ the derivative $\left(\lambda_{2}-\lambda_{1}\right)$ must be nonnegative at $t=0$ :

$$
0 \leqslant\left(\lambda_{2}-\lambda_{1} \dot{)}=\int_{0}^{\pi}\left(V_{1}(x)-V_{0}(x)\right)\left(y_{2}^{2}(x, 0)-y_{1}^{2}(x, 0)\right) d x .\right.
$$

By (20) and (23) the product is nonpositive and its integral is nonnegative. This is possible only when $V_{1}=V_{0}$ (except for the points $x=0, \frac{\pi}{2}, \pi$ ), i.e. the optimal $V_{0}$ must be a stepfunction with the only jump at $\frac{\pi}{2}$.

B) $\frac{\pi}{2}<x_{-}$(the case $x_{+}<\frac{\pi}{2}$ is similar). Now let

$$
V_{1}(x)=\left\{\begin{array}{lll}
V_{0}\left(\frac{\pi}{2}\right) & \text { on } & \left(0, x_{-}\right), \\
V_{0}\left(x_{+}\right) & \text {on } & \left(x_{-}, \pi\right) .
\end{array}\right.
$$


Then (23) remains valid and just as above we can prove that $V_{1}=V_{0}$. Our second choice is

$$
V_{2}(x)=\left\{\begin{array}{lll}
0 & \text { on } & \left(0, x_{-}\right) \\
M & \text { on } & \left(x_{-}, \pi\right)
\end{array}\right.
$$

From the definition of $x_{-}$and from (19) we obtain

$$
\begin{gathered}
\int_{0}^{x_{-}}\left(y_{2}^{2}(x, 0)-y_{1}^{2}(x, 0)\right) d x>0, \\
\int_{x_{-}}^{\pi}\left(y_{2}^{2}(x, 0)-y_{1}^{2}(x, 0)\right) d x<0 .
\end{gathered}
$$

This gives by the optimality of $V_{0}$ that

$$
\begin{aligned}
0 \leqslant\left(\lambda_{2}-\lambda_{1}\right)=\int_{0}^{\pi}\left(V_{2}(x)-V_{0}(x)\right)\left(y_{2}^{2}(x, 0)-y_{1}^{2}(x, 0)\right) d x \\
=-V_{0}\left(\frac{\pi}{2}\right) \cdot \int_{0}^{x_{-}}\left(y_{2}^{2}(x, 0)-y_{1}^{2}(x, 0)\right) d x \\
\quad+\left(M-V_{0}\left(x_{+}\right)\right) \int_{x_{-}}^{\pi}\left(y_{2}^{2}(x, 0)-y_{1}^{2}(x, 0)\right) d x
\end{aligned}
$$

which is only possible when $V_{0}\left(\frac{\pi}{2}\right)=0$ and $M=V_{0}\left(x_{+}\right)$, i.e. the optimal $V_{0}$ must be of the form $V_{2}$. But this is impossible for large $M$. Indeed, the second eigenfunction of the potential $V_{2}$ can be expressed by

$$
y_{2}(x)= \begin{cases}c \sin \left(\sqrt{\lambda_{2}} x\right) & \text { on }\left(0, x_{-}\right), \\ d \sin \left(\sqrt{\lambda_{2}-M}(\pi-x)\right) & \text { on }\left(x_{-}, \pi\right) .\end{cases}
$$

The only inner zero $x_{0}$ must lie between $x_{-}$and $x_{+}$, so on $\left(0, \frac{\pi}{2}\right), y_{2} \neq 0$. This is only possible when $\sqrt{\lambda_{2}} \frac{\pi}{2}<\pi$, i.e. when $\lambda_{2}<4$. Now for $M \geqslant 4$ we have $\lambda_{2}-M<0$ and then $0=y_{2}\left(x_{0}\right)=d \sin \left(\sqrt{\lambda_{2}-M}\left(\pi-x_{0}\right)\right)$ is impossible. The contradiction shows that case B) does not occur for $M \geqslant 4$. Summing up the above considerations we have seen that for $M \geqslant 4$ the optimal $V_{0}$ must have the form

$$
V_{0}=\left\{\begin{array}{lll}
0 & \text { on } \quad\left(0, \frac{\pi}{2}\right), \\
m & \text { on } \quad\left(\frac{\pi}{2}, \pi\right),
\end{array} \text { or } \quad V_{0}=\left\{\begin{array}{lll}
m & \text { on } & \left(0, \frac{\pi}{2}\right), \\
0 & \text { on } & \left(\frac{\pi}{2}, \pi\right),
\end{array}\right.\right.
$$

with some $m \geqslant 0$. The two potentials have the same eigenvalues so we deal only with the first form of $V_{0}$. In this case an eigenfunction corresponding to the eigenvalue $\lambda$ can be expressed as

$$
y(x)= \begin{cases}c \sin (\sqrt{\lambda} x) & \text { on }\left(0, \frac{\pi}{2}\right), \\ d \sin (\sqrt{\lambda-M}(\pi-x)) & \text { on }\left(\frac{\pi}{2}, \pi\right) .\end{cases}
$$

The constants $c, d$ have to be chosen such that $y(x)$ is $C^{1}$-smooth at $\frac{\pi}{2}$. This can be done if and only if the quotients $\frac{y^{\prime}}{y}$, counted in $\frac{\pi}{2}$ from both sides, are the same, i.e. when

$$
\sqrt{\lambda} \cot \left(\sqrt{\lambda} \frac{\pi}{2}\right)=-\sqrt{\lambda-M} \cot \left(\sqrt{\lambda-M} \frac{\pi}{2}\right) .
$$

The eigenvalues are the real solutions $\lambda$ of (24); we allow cases when both sides are infinite. But Lemma 2.1 states that in this case $\lambda_{2}-\lambda_{1}>3$ if $m>0$. Hence the 
optimal $V_{0}$ (where $\lambda_{2}-\lambda_{1}=3$ ) must be constant. This proves Theorem 1.1 in the case when the transition point $a$ is at $\frac{\pi}{2}$. Suppose finally that $0<a<\frac{\pi}{2}$ (the case $a>\frac{\pi}{2}$ is similar). Let

$$
V(x, t)=\left\{\begin{array}{lll}
t & \text { if } & x \in(0, a) \\
0 & \text { if } & x \in(a, \pi)
\end{array}\right.
$$

Then for $t=0$

$$
\left(\lambda_{2}-\lambda_{1} \dot{)}=\int_{0}^{a}\left(y_{2}^{2}(x, 0)-y_{1}^{2}(x, 0)\right) d x .\right.
$$

Here $y_{1}(x, 0)=\sqrt{\frac{2}{\pi}} \sin x, y_{2}(x, 0)=\sqrt{\frac{2}{\pi}} \sin 2 x$, so $\int_{0}^{\frac{\pi}{2}}\left(y_{2}^{2}-y_{1}^{2}\right)=0$. This means by (20) that

$$
\left(\lambda_{2}-\lambda_{1}\right)^{\cdot}=\int_{0}^{a}\left(y_{2}^{2}-y_{1}^{2}\right)<0
$$

so for small values $t>0$ the single-well potential $V(x, t)$ gives an eigenvalue gap $\lambda_{2}-\lambda_{1}<3$. The same potential is a good counterexample for $a=\pi$ while for $a=0$ we can choose, e.g.

$$
V=\left\{\begin{array}{l}
0 \text { on }\left(0, \frac{2 \pi}{3}\right), \\
t \text { on }\left(\frac{2 \pi}{3}, \pi\right),
\end{array}\right.
$$

if $t>0$ is small enough. Theorem 1.1 is proved.

Proof of Theorem 1.3. It is similar to the above proof. The eigenfunctions of (4)(5) are normalized by the rule

$$
\int_{0}^{\pi} u_{n}^{2}(x) \varrho(x) d x=1
$$

and such that $u_{n}(x)>0$ for small $x>0$. Then $u_{1}>0$ on $(0, \pi)$ and there exists $0<x_{0}<\pi$ such that $u_{2}>0$ on $\left(0, x_{0}\right)$ and $<0$ on $\left(x_{0}, \pi\right)$. Further there exist values $0 \leqslant x_{-}<x_{0}<x_{+} \leqslant \pi$ such that

$$
u_{1}^{2}-u_{2}^{2}\left\{\begin{array}{lll}
<0 & \text { on } & \left(0, x_{-}\right) \cup\left(x_{+}, \pi\right), \\
>0 & \text { on } & \left(x_{-}, x_{+}\right),
\end{array}\right.
$$

both sets being nonempty; see Huang [7]. On the other hand if $\varrho_{0}, \varrho_{1}$ are singlebarrier densities and

$$
\varrho(x, t)=t \varrho_{1}(x)+(1-t) \varrho_{0}(x),
$$

then the derivative of $\lambda_{n}$ in $t$ is

$$
\dot{\lambda}_{n}=-\lambda_{n} \int_{0}^{\pi}\left(\varrho_{1}(x)-\varrho_{0}(x)\right) u_{n}^{2}(x, t) d x ;
$$

see Keller 9 or Huang [7. Consequently,

$$
\left(\frac{\lambda_{2}}{\lambda_{1}}\right)^{\cdot}=\frac{\lambda_{2}}{\lambda_{1}} \int_{0}^{\pi}\left(\varrho_{1}(x)-\varrho_{0}(x)\right)\left(u_{1}^{2}(x, t)-u_{2}^{2}(x, t)\right) d x .
$$

For $M>1$ denote

$$
A_{M}=\left\{\varrho: \frac{1}{M} \leqslant \varrho \leqslant M, \varrho \text { is single-barrier with transition point at } \frac{\pi}{2}\right\} .
$$


Since the minimum of $\lambda_{1}$ over $A_{M}$ must be positive, there exists an optimal $\varrho_{0} \in$ $A_{M}$ giving the minimal ratio $\frac{\lambda_{2}}{\lambda_{1}}$ over $A_{M}$. We analyze some cases like above. If $x_{-} \leqslant \frac{\pi}{2}<x_{+}$, then for

$$
\varrho_{1}=\left\{\begin{array}{l}
\varrho_{0}\left(x_{-}\right) \text {on }\left(0, \frac{\pi}{2}\right), \\
\varrho_{0}\left(x_{+}\right) \text {on }\left(\frac{\pi}{2}, \pi\right) ;
\end{array}\right.
$$

we get $\left(\varrho_{1}-\varrho_{0}\right)\left(u_{1}^{2}-u_{2}^{2}\right) \leqslant 0$ on $(0, \pi)$; hence (for $t=0$ )

$$
0 \leqslant\left(\frac{\lambda_{2}}{\lambda_{1}}\right)=\frac{\lambda_{2}}{\lambda_{1}} \int_{0}^{\pi}\left(\varrho_{1}-\varrho_{0}\right)\left(u_{1}^{2}-u_{2}^{2}\right)
$$

which is possible only when $\varrho_{1}=\varrho_{0}$ (except for $x=0, \frac{\pi}{2}$ and $\pi$ ). The case $x_{+}=\frac{\pi}{2}$ is similar. If $\frac{\pi}{2}<x_{-}$, then analogously we get

$$
\varrho_{0}=\varrho_{2}=\left\{\begin{array}{lll}
M & \text { on } & \left(0, x_{-}\right) \\
\frac{1}{M} & \text { on } & \left(x_{-}, \pi\right)
\end{array}\right.
$$

In this case we have

$$
u_{2}=\left\{\begin{array}{l}
c \sin \left(\sqrt{\lambda_{2} M} x\right) \text { on }\left(0, x_{-}\right), \\
d \sin \left(\sqrt{\lambda_{2} \frac{1}{M}}(\pi-x)\right) \text { on }\left(x_{-}, \pi\right) .
\end{array}\right.
$$

The only zero of $u_{2}$ lies between $x_{-}$and $x_{+}$. Since there are no zeros on $\left(0, x_{-}\right]$,

$$
\sqrt{\lambda_{2} M} x_{-}<\pi, \text { i.e. } \quad M<\frac{1}{\lambda_{2}} \cdot\left(\frac{\pi}{x_{-}}\right)^{2} .
$$

Since there is a zero on $\left(x_{-}, \pi\right)$ we get

$$
\sqrt{\lambda_{2} \frac{1}{M}}\left(\pi-x_{-}\right)>\pi, \quad \text { i.e. } \quad M<\lambda_{2}\left(\frac{\pi-x_{-}}{\pi}\right)^{2} .
$$

Multiplying these inequalities leads to a contradiction

$$
M^{2}<\left(\frac{\pi-x_{-}}{x_{-}}\right)^{2}<1
$$

The case $x_{+}<\frac{\pi}{2}$ is similar. This shows that (after multiplying by an appropriate constant) an optimal $\varrho_{0}$ must have the form

$$
\varrho_{0}=\left\{\begin{array}{ll}
1 & \text { on }\left(0, \frac{\pi}{2}\right), \\
m & \text { on }\left(\frac{\pi}{2}, \pi\right),
\end{array} \quad \text { or } \varrho_{0}= \begin{cases}m & \text { on }\left(0, \frac{\pi}{2}\right), \\
1 & \text { on }\left(\frac{\pi}{2}, \pi\right),\end{cases}\right.
$$

with some $m \geqslant 1$. We analyze only the first case. An eigenfunction of $\varrho_{0}$ is of the form

$$
u=\left\{\begin{array}{l}
c \sin (\sqrt{\lambda} x) \quad \text { on }\left(0, \frac{\pi}{2}\right) \\
d \sin (\sqrt{\lambda m}(\pi-x)) \text { on }\left(\frac{\pi}{2}, \pi\right) .
\end{array}\right.
$$

The condition of being in $C^{1}$ at $\frac{\pi}{2}$ gives

$$
\sqrt{\lambda} \cot \left(\sqrt{\lambda} \frac{\pi}{2}\right)=-\sqrt{\lambda m} \cot \left(\sqrt{\lambda m} \frac{\pi}{2}\right) .
$$


The eigenvalues are the positive solutions of (29); we allow that both sides be infinite. Using the notation $t=\sqrt{\lambda} \frac{\pi}{2}>0, d=\sqrt{m} \geqslant 1$ this becomes

$$
\tan t=-\frac{1}{d} \tan (t d)
$$

It is enough to prove that the first two positive solutions of (30) satisfy $t_{2}>2 t_{1}$ unless $d=1$. This is exactly what is stated in Lemma 2.2, so Theorem 1.2 is proved if $a=\frac{\pi}{2}$ is the transition point. Finally we can prove that $a \neq \frac{\pi}{2}$ does not yield $\lambda_{2} \geqslant 4 \lambda_{1}$ in the same way as Theorem 1.1: if

$$
\varrho=\left\{\begin{array}{lll}
1 & \text { on } & (0, a) \\
c & \text { on } & (a, \pi)
\end{array}\right.
$$

and $0<a<\pi, a \neq \frac{\pi}{2}$, then for small $|c-1| \neq 0$ we have $\lambda_{2}<4 \lambda_{1}$ if $c>1$ and

$a>\frac{\pi}{2}$ or if $c<1$ and $a<\frac{\pi}{2}$. These densities are also good counterexamples for $a=0$ resp. $a=\pi$. Theorem 1.3 is proved.

\section{ACKNOWLEDGEMENT}

The author is indebted to the referee for calling his attention to the paper [1] of Abramovich.

\section{REFERENCES}

1. S. Abramovich, The gap between the first two eigenvalues of a one-dimensional Schrödinger operator with symmetric potential, Proc. Amer. Math. Soc. 111 (1991), 451-453. MR 92f:34077

2. M.S. Ashbaugh and R. Benguria, Best constant for the ratio of the first two eigenvalues of one-dimensional Schrödinger operators with positive potential, Proc. Amer. Math. Soc. 99 (1987), 598-599. MR 88e:34039

3. with Dirichlet boundary conditions and positive potentials, Commun. Math. Phys. 124 (1989), 403-415. MR 91c:34114

4. - Optimal lower bound for the gap between the first two eigenvalues of one-dimensional Schrödinger operators with symmetric single-well potentials, Proc. Amer. Math. Soc. 105 (1989), 419-424. MR 89f:81028

5. —_ Eigenvalue ratios for Sturm-Liouville operators, J. Diff. Equations 103 (1993), 205219. MR 94c:34125

6. R.D. Gentry and D.O. Banks, Bound for functions of eigenvalues of vibrating systems, J. Math. Anal. Appl. 51 (1975), 100-128. MR 51:8528

7. M.-J. Huang, On the eigenvalue ratio with vibrating strings, Proc. Amer. Math. Soc. 127 (1999), no. 6, 1805-1813. MR 99i:34119

8. Y.-L. Huang and C.K. Law, Eigenvalue ratios for the regular Sturm-Liouville system, Proc. Amer. Math. Soc. 124 (1996), 1427-1436. MR 96g:34044

9. J.B. Keller, The minimum ratio of two eigenvalues, SIAM J. Appl. Math. 31 (1976), 485-491. MR 54:10737

10. R. Lavine, The eigenvalue gap for one-dimensional convex potentials, Proc. Amer. Math. Soc. 121 (1994), 815-821. MR 94i:35144

11. T.J. Mahar and B.E. Willner, An extremal eigenvalue problem, Comm. Pure Appl. Math. 29 (1976), 517-529. MR 54:13201

Department for Mathematical Analysis, Institute of Mathematics, Technical University of Budapest, H 1111 Budapest, Múegyetem RKP. 3-9, Hungary

E-mail address: horvath@math.bme.hu 